\title{
Reentrant transitions in colloidal or dusty plasma bilayers
}

\author{
René Messina* and Hartmut Löwen才 \\ Institut für Theoretische Physik II, Heinrich-Heine-Universität Düsseldorf, \\ Universitätsstrasse 1, D-40225 Düsseldorf, Germany
}

(Dated: November 6, 2018)

\begin{abstract}
The phase diagram of crystalline bilayers of particles interacting via a Yukawa potential is calculated for arbitrary screening lengths and particle densities. Staggered rectangular, square, rhombic and triangular structures are found to be stable including a first-order transition between two different rhombic structures. For varied screening length at fixed density, one of these rhombic phases exhibits both a single and even a double reentrant transition. Our predictions can be verified experimentally in strongly confined charged colloidal suspensions or dusty plasma bilayers.
\end{abstract}

PACS numbers: 68.65.Ac, 82.70.Dd, 52.27.Lw

Confined systems exhibit structural and dynamical behavior very much different from the corresponding bulk state [1, 2]. In particular freezing is strongly affected by the presence of a planar wall. In equilibrium, solidification near walls can occur at thermodynamic conditions where the bulk is still fluid (so called "prefreezing") 3, 4]. In nonequilibrium, the wall may act as a center of heterogeneous nucleation [5] in order to initiate crystal growth [6]. A system confined between two parallel planar walls exhibits various layered crystalline states at low temperature if the plate distance gets comparable to the mean interparticle distance. For hard spheres between hard plates, geometric packing considerations lead to the stability of different crystalline lattices including multiple square and hexagonal layers [7] as well as buckled [8], rhombic [8, 9] and prism superlattices [10]. On the other hand, for pure coulombic systems like (classical) electrons in quantum wells 11] or trapped ions 12], several crystalline bilayer-structures were reported [13].

Most of our experimental knowledge of freezing in confining slit-like geometry is based on real-space measurements of mesoscopic model systems such as charged colloidal suspensions between glass plates [7, 10] or of multilayers of highly charged dusty plasmas [14]. The actual interaction between these mesoscopic "macroions" is neither hard-sphere like nor pure coulombic but is described by an intermediate screened Coulomb or Yukawa pair potential [15, 16] due to the screening via additional microions in the system. The screening length can be tailored by changing the microion concentration: for charged colloids, salt ions are conveniently added to the aqueous suspensions; the complex plasma, on the other hand, consists of electrons and impurity ions.

In the present letter, we study the stability of different crystal lattices in bilayers of Yukawa particles as motivated by the experimental model systems. The zerotemperature phase diagram is calculated for arbitrary screening lengths and particle densities [17]. We find a variety of different staggered solid lattices to be stable which are separated by either first or second-order phase transitions. The two known extreme limits of zero or infinite screening length corresponding to hard-spheres [9] and the plasma [13, 18] are recovered. For intermediate screening lengths, the phase behavior is strikingly different from a simple interpolation between these two limits. First, there is a first-order coexistence between two different staggered rhombic lattices differing in their relative shift of the two unit cells. Second, one of these staggered rhombic phases exhibits a novel reentrant effect for fixed density and varied screening length. Depending on the density, the reentrant transition can proceed via a staggered square or a staggered triangular solid including even a double reentrant transition of the rhombic phase. All our theoretical predictions can in principle be verified in real-space studies of confined charged suspensions or dusty plasmas.

In detail, our system consists of two layers containing in total $N$ particles in the $(x, y)$ plane. The total area density of the two layers is $\rho=N / A$ with $A$ denoting the system area in the $(x, y)$ plane. The distance $D$ between the layers in the $z$ direction is prescribed by the external potential confining the system. The particles are interacting via the Yukawa pair potential

$$
V(r)=V_{0} \frac{\exp (-\kappa r)}{\kappa r}
$$

where $r$ is the central separation. The inverse screening length $\kappa$ which governs the range of the interaction is given in terms of the micro-ion concentration by Debye-Hückel screening theory. The energy amplitude $V_{0}=Z^{2} \exp (2 \kappa R) \kappa / \epsilon(1+\kappa R)^{2}$ scales with the square of the charges $Z$ of the particles of physical hard core radius $R[19]$ reduced by the dielectric $\epsilon$ permittivity of the solvent ( $\epsilon=1$ for the dusty plasma). Typically, $Z$ is of the order of $100-100000$ elementary charges such that $V(r)$ at typical interparticle distances can be much larger than the thermal energy $k_{B} T$ at room temperature justifying formally zero-temperature calculations. Then the energy scale is set by $V_{0}$ alone and phase transitions in large bilayer systems are completely determined by two dimensionless parameters, namely the reduced layer density $\eta=\rho D^{2} / 2$ and the relative screening strength $\lambda=\kappa D$. 
For zero temperature, the stable state is solid but different crystalline structures of the bilayers are conceivable. As possible candidate structures we assume that the two two-dimensional periodic lattices in the bilayers are the same, have a simple unit cell and are shifted relative to each other in the lateral direction by a displacement vector c. If the two layers are labeled with $A$ and $B$, the particle positions in the $(x, y)$ plane of the two layers are given by

$$
\begin{aligned}
& \mathbf{R}_{A}(m, n)=m \mathbf{a}_{\mathbf{1}}+n \mathbf{a}_{\mathbf{2}}, \\
& \mathbf{R}_{B}(m, n)=m \mathbf{a}_{\mathbf{1}}+n \mathbf{a}_{\mathbf{2}}+\mathbf{c},
\end{aligned}
$$

where $\mathbf{a}_{\mathbf{1}}$ and $\mathbf{a}_{\mathbf{2}}$ are the primitive vectors of the twodimensional lattice and $m, n$ are integers. The total internal energy $U$ is obtained by the double lattice sum

$$
\begin{aligned}
U= & \frac{1}{2} \sum_{\mathbf{R}_{A} \neq \mathbf{R}_{A}^{\prime}} V\left(\left|\mathbf{R}_{A}-\mathbf{R}_{A}^{\prime}\right|\right)+\frac{1}{2} \sum_{\mathbf{R}_{B} \neq \mathbf{R}_{B}^{\prime}} V\left(\left|\mathbf{R}_{B}-\mathbf{R}_{B}^{\prime}\right|\right) \\
& +\sum_{\mathbf{R}_{A}, \mathbf{R}_{B}} V\left(\left[\left|\mathbf{R}_{A}-\mathbf{R}_{B}\right|^{2}+D^{2}\right]^{1 / 2}\right) .
\end{aligned}
$$

In the limit $N \rightarrow \infty$, the stable crystalline structure minimizes the total internal energy per particle $U / N$.

We have minimized $U / N$ with respect to $\mathbf{a}_{\mathbf{1}}, \mathbf{a}_{\mathbf{2}}$ and $\mathbf{c}$ under the constraint of prescribed density $\eta$ for given $\lambda$ mapping out the phase diagram in the $(\eta \lambda)$ plane. As a result, five typical staggered lattice structures turn out to minimize $U / N$ for different $\eta$. Adopting the notation developed for plasma bilayers [18], we label them by I, II, III, IV, and V. As summarized in Table I] phase I is the staggered rectangular crystal with a fixed aspect ratio $a_{2} / a_{1}$ of $\sqrt{3}$; phase II is also staggered rectangular but with a different aspect ratio $\gamma$ interpolating continuously between phase I and the staggered-square phase III where $a_{2} / a_{1}=1$. The staggered rhombic phase IV has two non-orthogonal lattice unit vectors $\left(\mathbf{a}_{1}\right.$ and $\left.\mathbf{a}_{2}\right)$ forming an angle $\theta$ and contains a general lateral shift $\mathbf{c}=\alpha\left(\mathbf{a}_{1}+\mathbf{a}_{2}\right)$ between the two rhombic lattices. In fact, we find two possibilities for $\alpha$ defining two variants of stable rhombic phases which we call IVA and IVB. For IVA, $\alpha=1 / 2$ while $\alpha<1 / 2$ for IVB. Finally, phase V

TABLE I: Structure and parameters of the different staggered bilayer crystals. $\mathbf{a}_{1}$ is set to $\left(a_{1}, 0\right)$ where $a_{1}$ is the nearest intralayer distance between particles. For phase II, $\gamma=a_{2} / a_{1}$ is the aspect ratio. For phase IV, $\theta$ is the angle between $\mathbf{a}_{1}$ and $\mathbf{a}_{2}$, and $\alpha$ is a free parameter characterizing the relative lateral interlattice shift $\mathbf{c}$.

\begin{tabular}{lccc}
\hline \hline Phase & $\mathbf{a}_{2} / a_{1}$ & $\mathbf{c}$ & $\rho a_{1}^{2} / 2$ \\
\hline I. Rectangular & $(0, \sqrt{3})$ & $\left(\mathbf{a}_{1}+\mathbf{a}_{2}\right) / 2$ & $1 / \sqrt{3}$ \\
II. Rectangular & $(0, \gamma)$ & $\left(\mathbf{a}_{1}+\mathbf{a}_{2}\right) / 2$ & $\gamma$ \\
III. Square & $(0,1)$ & $\left(\mathbf{a}_{1}+\mathbf{a}_{2}\right) / 2$ & 1 \\
IV. Rhombic & $(\cos \theta, \sin \theta)$ & $\left(\mathbf{a}_{1}+\mathbf{a}_{2}\right) \alpha$ & $1 / \sin \theta$ \\
V. Triangular & $(1 / 2, \sqrt{3} / 2)$ & $\left(\mathbf{a}_{1}+\mathbf{a}_{2}\right) / 3$ & $2 / \sqrt{3}$ \\
\hline \hline
\end{tabular}
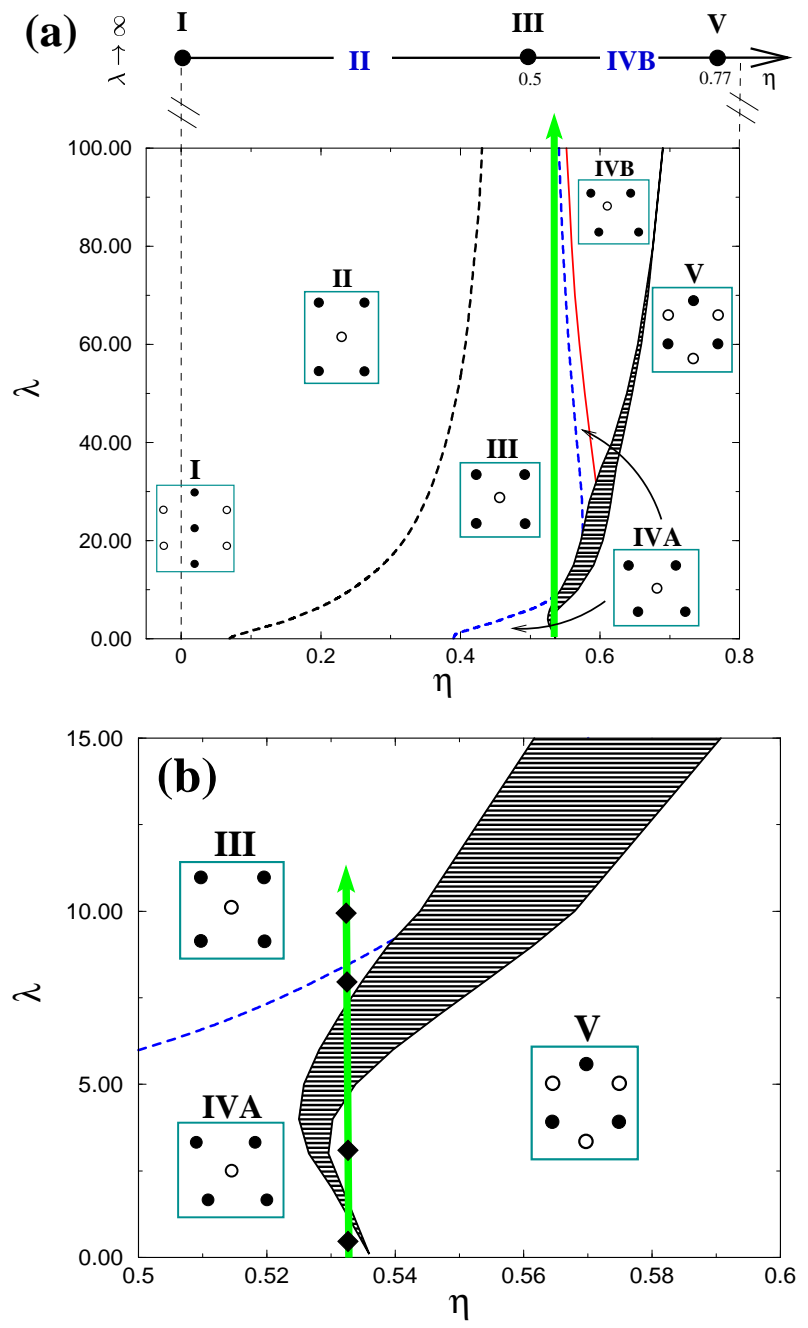

FIG. 1: Phase diagram of the Yukawa bilayer in the $(\eta, \lambda)$ plane. (a) The hard sphere limit $\lambda \rightarrow \infty$ is sketched on top. The dashed (solid) lines denote continuous (discontinuous) transitions. The filled region corresponds to the coexistence domain of phases IV and V. The vertical arrow indicates the double reentrant behavior of phase IVA. The insets show the lattice geometries, where the filled (open) circles correspond to the lower (upper) layer. (b) Magnification of (a) showing a reentrant behavior of phase IVA occurring at moderate $\lambda$. The four diamonds along the arrow indicate state points which were investigated by computer simulation at finite temperatures.

is a staggered triangular crystal. Both phases III and V can be considered as special cases of the rhombic phase IV; the former has $\theta=\pi / 2$ and $\alpha=1 / 2$ while the latter is characterized by $\theta=\pi / 3$ and $\alpha=1 / 3$.

The result for the phase diagram for a wide range of screening strengths $(0 \leq \lambda \leq 100 ; \lambda \rightarrow \infty)$ and densities $(0 \leq \eta \leq 0.8)$ is shown in Fig. 1 At very low screening $\lambda$, we recover the known plasma limit 18, with our labeling of the phases being in line with their sequence for increasing density $\eta$. Phase I has a finite but extremely 
small density region of stability up to $\eta=3.6 \times 10^{-5}$ at $\lambda=0$ [18]. For finite $\lambda$, the $\mathrm{I} \rightarrow \mathrm{II}$ transition stays second-order and occurs at even smaller densities which decrease monotonically to zero as a function of $\lambda$ until the hard-sphere limit $\lambda \rightarrow \infty$ is reached. In this latter case $a_{1}$ is playing the role of an effective particle diameter. This is sketched by the vertical line in Fig. 1 The II $\rightarrow$ III transition is second-order and the transition densities increase drastically with growing $\lambda$ and interpolating monotonically between the plasma and hard sphere limit. More details of the I $\rightarrow$ II $\rightarrow$ III transition scenario are depicted in Fig. 2] where the aspect ratio $\gamma$ of phase II is shown versus $\eta$ for different $\lambda$. Phases I and III correspond to $\gamma=\sqrt{3}$ and $\gamma=1$, respectively. As can be clearly deduced from Fig. 2 the aspect ratio $\gamma$ interpolates continuously as a function of $\eta$ between $\sqrt{3}$ and 1 for any $\lambda$ such that both the I $\rightarrow$ II and the II $\rightarrow$ III transitions are second-order. In the hard sphere limit, $\gamma$ approaches $\gamma_{h s}=-2 \eta+\sqrt{4 \eta^{2}+3}$ continuously which is also shown in Fig. 2

Novel effects are observed for the III $\rightarrow$ IV $\rightarrow$ V transitions. First, for small $\lambda$ the III $\rightarrow \mathrm{V}$ transition proceeds via a IVA phase, the former being second and the latter first-order. For $\lambda \approx 8$, however, there is a strong firstorder transition directly from III to $\mathrm{V}$ with a large density jump as determined by Maxwell's construction [20]. For even higher screening, $\lambda \gtrsim 30$, the III $\rightarrow \mathrm{V}$ transition happens via the cascade III $\rightarrow$ VIA $\rightarrow$ IVB $\rightarrow$ V. The stability range of the IVA phase becomes smaller for increasing $\lambda$ shrinking to zero in the hard sphere limit. Details of the III $\rightarrow$ V transition scenario can be detected via the order parameters $\sin \theta$ and $\alpha$ of the lattice min-

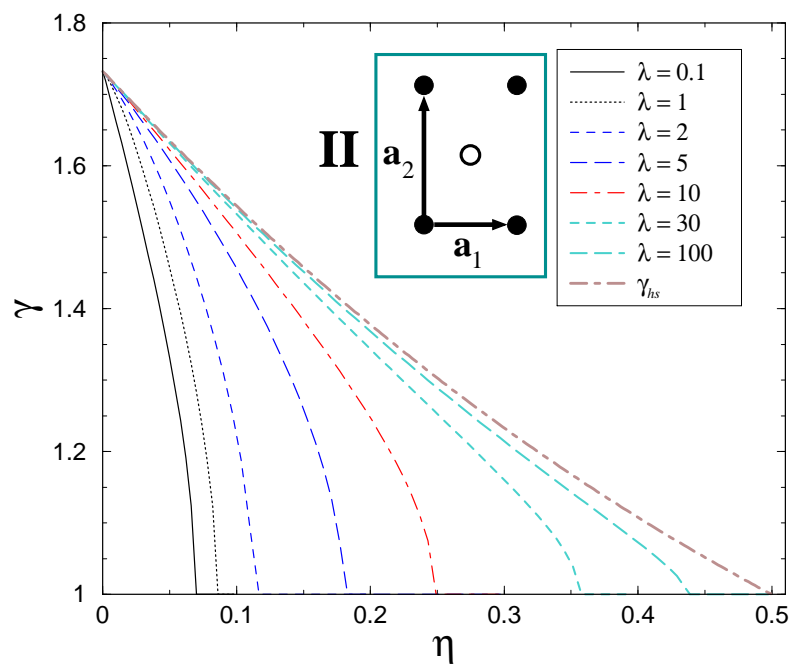

FIG. 2: Aspect ratio $\gamma=a_{2} / a_{1}$ for phase II versus density $\eta$ for different screening strengths $\lambda$. The hard sphere case $\gamma_{h s}$ is also shown. The lattice geometry is shown as an inset, where the filled (open) circles correspond to the lower (upper) layer.
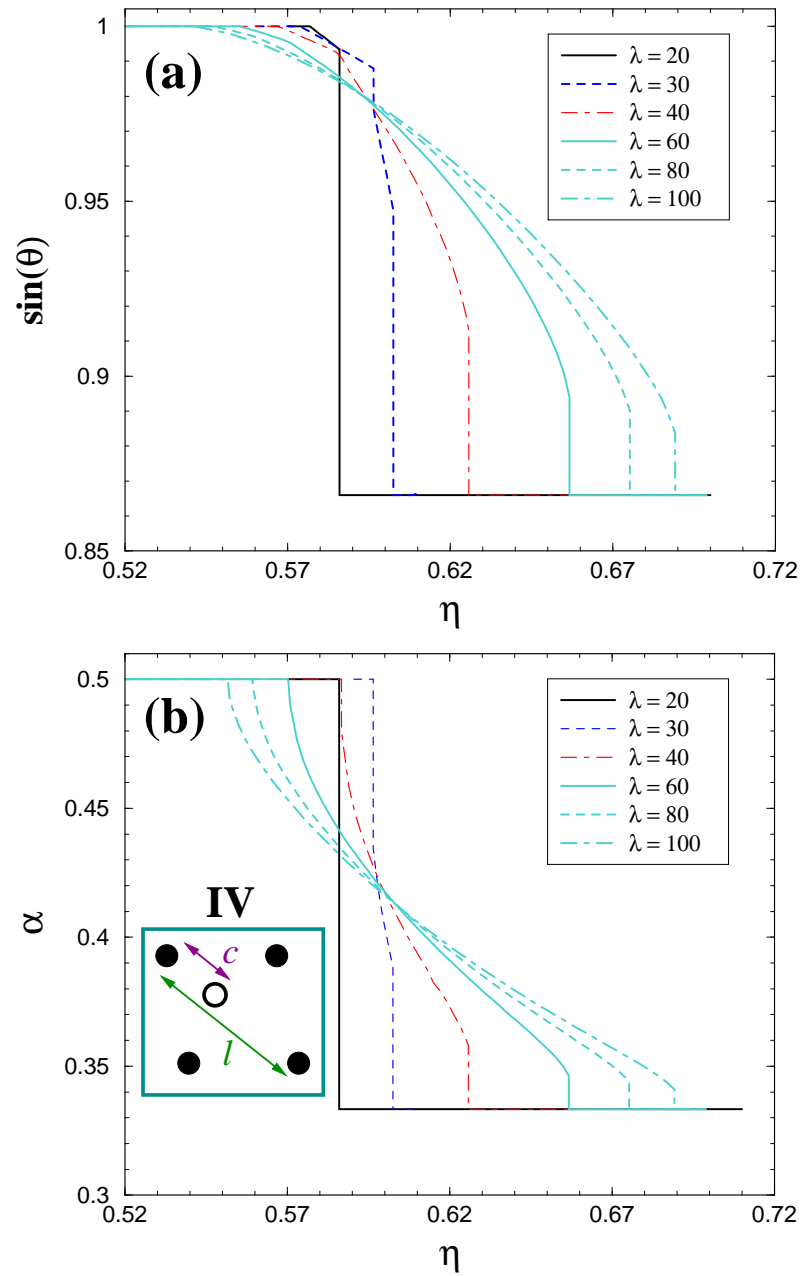

FIG. 3: (a) Sine of the angle $\theta$ and (b) the relative shift parameter $\alpha=c / \ell$ (with $\ell=\left|\mathbf{a}_{1}+\mathbf{a}_{2}\right|$ ) versus density regarding the III $\rightarrow \mathrm{IV} \rightarrow \mathrm{V}$ transition scenario for different $\lambda$. The insets show the lattice geometry of phase IV.

imizing the total potential energy at prescribed density $\eta$. Plotting $\sin \theta$ and $\alpha$ versus $\eta$ reveals the order of the transitions (see Fig. 3): A cusp, which is found for the III $\rightarrow$ IVA transformation, implies a second-order transition. All other transitions are first-order as signaled by discontinuous jumps in at least one of these order parameters. The corresponding coexistence density gap is not shown in Fig. 3 but included in Fig. 1(a). Across the IVA $\rightarrow$ IVB transition the order parameter jump is small yielding a tiny density gap which can not be resolved in Fig. 1(a).

Our most striking result is reentrant behavior of the IVA phase at fixed density upon varying $\lambda$ as indicated in Fig. 1 by the vertical arrow. For $0.5<\eta<0.525$, there is reentrance of the VIA phase via the III phase. The full sequence over the whole range of $\lambda$ is IVA $\rightarrow$ $\mathrm{III} \rightarrow$ IVA $\rightarrow$ IVB. For $0.530<\eta<0.536$ there is even a double reentrant behavior of the VIA phase via 
the sequence IVA $\rightarrow \mathrm{V} \rightarrow \mathrm{IVA} \rightarrow \mathrm{III} \rightarrow \mathrm{IVA} \rightarrow \mathrm{IVB}$. This rich scenario is due to a subtle interplay of the range of the interaction in conjunction with the different bilayer lattice structures. Finally, at finite temperatures $T$, we performed extensive Monte-Carlo computer simulations with 800 particles in a rectangular-shaped box periodically-repeated in $x$ and $y$ direction and with hard walls of distance $D$ in $z$-direction allowing fluctuating $z$-positions of the particles ("buckling"). For fixed $\eta=0.533$, we investigated four states at $\lambda=0.5,3.0,8.2$, 10 [see the four diamonds along the arrow in Fig. 11(b)] for different $T$ up to melting. The melting point is detected via a modified Lindemann-type criterion involving differences of mean-square displacements of nearest-neighbors 13. We confirm that the reentrant behavior is stable with respect to increasing $T$ up to melting.

In summary, we have calculated the full phase diagram for a Yukawa bilayer at zero temperature by lattice sum minimizations. A competition between three length scales, namely the bilayer distance $D$, the averaged particle distance $\rho^{-1 / 2}$, and the range $1 / \kappa$ of the interaction, induces a rich phase behavior which is different from a simple interpolation of the extreme limits of the confined plasma and the hard sphere system. We predict a coexistence of two different rhombic phases at finite screening and a single and double reentrant scenario for one of the rhombic phases for varied "softness" of the interaction. These effects are in principle detectable in real-space experiments of charged colloidal suspensions confined between plates and in layers of dusty plasmas by tuning the screening strength via the microion concentration. The reentrant effect as obtained here in equilibrium should also manifest itself as an interesting fingerprint in nonequilibrium situations. For example, bilayer crystal nucleation and growth could be greatly stimulated via structures which are energetically close to the stable ones 21]. Soft particle interactions different from the Yukawa type of Eq. (1), as e.g. inverse power potentials where $V(r) \propto r^{-n}$, will lead to similar reentrant effects as long as the softness of the potential (e.g. the exponent $n$ ) is varied. Different realizations of soft interactions occur in sterically-stabilized colloids, in spherical block-copolymer micelles and in star polymers and dendrimers, where the softness of the effective interaction can be tuned by the length and grafting density of the polymer chains or the solvent quality 22]. Hence the reentrant scenario should also occur in foam films containing polymer bilayers [23]. Finally, for a general external potential confining the particles to layers, the bilayer distance $D$ is not prescribed but the system will minimize its total energy realizing an optimal $D$. In this case, second-order phase transitions will still be described in terms of scaled parameters. This implies a universal behavior of our bilayer phase diagram. In a general ex- ternal potential, however, the system has the additional possibility to split into tri- and higher order multilayers. This can happen either discontinuously or continuous via merging prism phases. Details have to be explored in future studies.

We thank C. N. Likos and M. Schmidt for helpful discussions, and the DFG (SFB TR6) for financial support.

* Electronic address: messina@thphy.uni-duesseldorf.de

$\dagger$ Electronic address: hlowen@thphy.uni-duesseldorf.de

[1] K. Binder, Journal of Non-Equilibrium Thermodynamics 23, 1, 1998.

[2] C. Bechinger and E. Frey, J. Phys. Condens. Matter 13, R321 (2001).

[3] J. E. Hug., F. van Swol, and C. F. Zukoski, Langmuir, 11, 111 (1997).

[4] M. Heni, H. Löwen, Phys. Rev. Lett. 85, 3668 (2000).

[5] M. Würth et al., Phys. Rev. E 52, 6415 (1995).

[6] K. P. Velikov et al., Science 296, 106 (2002).

[7] C. A. Murray, W. O. Sprenger, and R. A. Wenk, Phys. Rev. B 42, 688 (1990).

[8] P. Pieranski and L. Strzelecki, Phys. Rev. Lett. 50, 331 (1983).

[9] M. Schmidt, H. Löwen, Phys. Rev. Lett. 76, 4552 (1996); Phys. Rev. E 55, 7228 (1997).

[10] S. Neser et al., Phys. Rev. Lett. 79, 2348 (1997).

[11] G. J. Kalman, Z. Donko, K. I. Golden, Contrib. Plasma Phys. 41, 191 (2001).

[12] M. G. Raizen et al., Phys. Rev. A 45, 6493 (1992).

[13] I. V. Schweigert, V. A. Schweigert and F. M. Peeters, Phys. Rev. Lett. 82, 5293 (1999); Phys. Rev. B 60, 14665 (1999).

[14] M. Zuzic et al., Phys. Rev. Lett. 854064 (2000).

[15] A recent justification for the Yukawa interaction in dusty plasmas can be found in: S. Nunomura et al., Phys. Rev. Lett. 89, 035001 (2002).

[16] M. O. Robbins, K. Kremer, G. S. Grest, J. Chem. Phys. 88, 3286 (1988).

[17] For moderate screening strength $\lambda$, the bilayer phase diagram (obtained by Monte Carlo simulations) was already reported in Ref. 13] but without the reentrance effect, the density jump across first-order transitions and the stable IVB phase.

[18] G. Goldoni and F. M. Peeters, Phys. Rev. B 53, 4591 (1996).

[19] We neglect the non-overlap condition $r>2 R$ in Eq. (1) since charged particles are typically not at contact.

[20] Strictly speaking, volume terms of the microions will also contribute to the density jumps, but these terms are only relevant for strongly deionized systems, see R. van Roij and J.-P. Hansen, Phys. Rev. Lett. 79, 3082 1998. For high salinity, these terms will shrink the density jumps, see H. Graf and H. Löwen, Phys Rev. E 57, 5744 (1998).

[21] T. Palberg, J. Phys. Condens. Matter 11, R323 (1999).

[22] C. N. Likos et al., Phys. Rev. Lett. 80, 4450 (1998).

[23] B. Kolaric, W. Jaeger and R. von Klitzing, J. Phys. Chem. B, 104, 5096 (2000). 